\title{
A Novel Hand-Specific External Fixator
}

\section{Virak Tan*}

Institute for Hand and Arm Surgery, Harrison, NJ, USA

\section{Introduction}

Finger injuries are common in sports, falls and occupational trauma. Although most of these injuries can adequately be treated with traditional means including closed reduction, splinting or internal fixation, there are some that are difficult to effectively manage, due to the severity of bony comminution and/or joint involvement. In such cases, internal fixation might not be preferred or possible, especially if there is soft tissue compromise. Thus, management with an external fixator is an attractive alternative.

While finger external fixators have been described, often they are not specific to the hand. Because of the varied injury patterns that occur in the fingers, a versatile and adjustable external fixator would be advantageous to treat these conditions. The DigiFix ${ }^{\oplus}$ External Fixator (Virak Orthopedics LLC, Harrison, NJ, Figure 1) was specifically designed to be low-profile for the hand, and its unique features allow it to function in either the dynamic or static state; and in distraction, nondistracted or compression mode. This external fixator attaches to the phalanges on the sides of the finger with smooth stainless steel K-wires. In the dynamic mode, the axis of rotation of the fixator is aligned to the axis of rotation of the joint, and the distal skeletal fixation is collinear to the mid-axis of the bone to maintain concentric joint reduction. Joint distraction is achieved by elongating the Diamond portion of the device with parallel pliers. The DigiFix ${ }^{\circ}$ can be also be used in the static mode in cases of phalangeal shaft fractures or joint fusions. Compression, if desired, is generated by expanding the Diamond with a spreader instrument.

The DigiFix ${ }^{\oplus}$ is indicated for the treatment of: 1) complex fracturedislocation/subluxation, unstable dislocation, and pilon fracture of the interphalangeal (IP) joint; 2) contracture of the proximal interphalangeal (PIP) joint; 3) phalangeal shaft fracture; and 4) interphalangeal joint arthrodesis. Contraindications include poor patient compliance, active infection of the finger, and severe osteoporosis whereby there is poor purchase of $\mathrm{K}$-wires into the phalanges. The purpose of this article is to report on the different uses of the DigiFix external fixator in hand trauma.

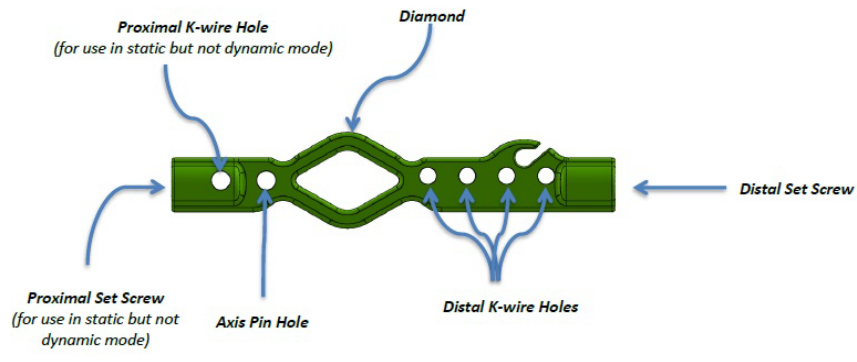

Figure 1: Diagram of the DigiFix® Bracket. In the dynamic mode, the Axis Pin Hole of the Bracket is aligned to the center of rotation of the joint, and the distal skeletal fixation is collinear to the mid-axis of the bone to maintain concentric joint reduction. Joint motion is permitted as the Bracket pivots about axis pin. For static mode, another K-wire is placed through the Proximal K-wire Hole as a second point of fixation in the proximal bone segment. All K-wires, except for one through the Axis Pin Hole, can be locked to the Bracket by tightening the set screws at the ends of the Bracket
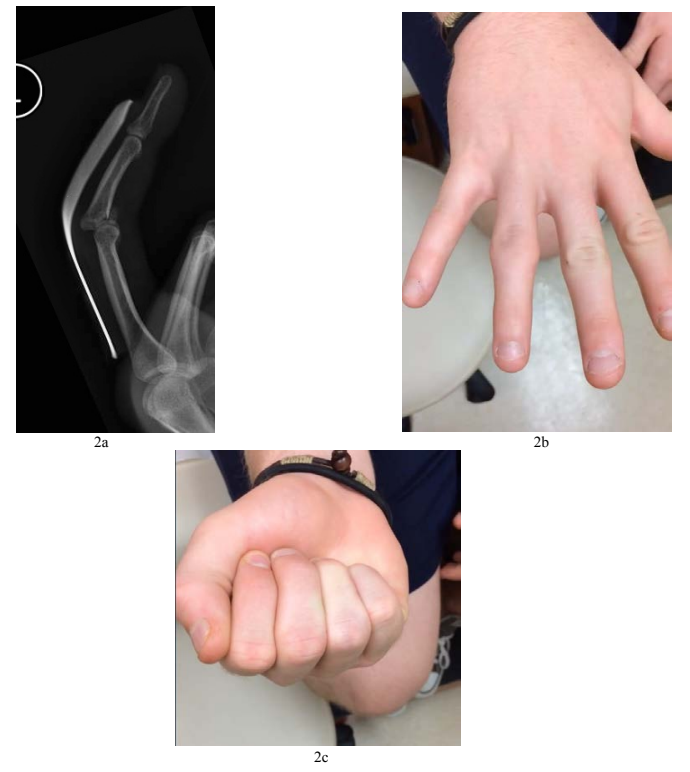

Figure 2: a) Lateral radiograph showing a PIP fracture-dislocation with $50 \%$ articular involvement. b \& c) Clinical photographs at 6 months post-op.

\section{Case 1: Proximal Interphalangeal Joint Fracture-Dislo- cation}

The patient is a 23 year old male who sustained an axial load to the tip of the right ring finger. He suffered a P2 volar base fracture with dorsal fracture-dislocation of the PIP joint (Figure 2a). The DigiFix ${ }^{\oplus}$ was applied in the dynamic mode and PIP motion was initiated immediately. The fixator was maintained for 5 weeks. At 6 months post-injury, the patient exhibited full motion and strength in the hand (Figure $2 \mathrm{~b}$ and $2 \mathrm{c}$ ).

\section{Case 2: Proximal Interphalangeal Joint Pilon Fracture}

The patient is a 48 year old woman who jammed her left ring finger. Radiographs showed an intra-articular pilon fracture (Figure 3a). She was taken to the operating room, where a K-wire was placed from dorsal to volar to secure the P2 base fragments. The DigiFix ${ }^{\circledR}$ was then applied in distraction across the PIP joint and initially set in the static mode (Figure 3b-3d). After 3.5 weeks, the fixator was dynamized by removing the most-proximal K-wire and joint motion was permitted. The external fixator was removed at 5.5 weeks. At 8 weeks post-injury,

*Corresponding author: Virak Tan, Institute for Hand and Arm Surgery, 620 Essex St., Harrison, NJ, USA, Tel: +19739474700; E-mail: hand_doc@yahoo.com

Received September 09, 2015; Accepted October 20, 2015; Published October 28 2015

Citation: Tan V (2015) A Novel Hand-Specific External Fixator. J Trauma Treat 4 269. doi:10.4172/2167-1222.1000269

Copyright: () 2015 Tan V. This is an open-access article distributed under the terms of the Creative Commons Attribution License, which permits unrestricted use, distribution, and reproduction in any medium, provided the original author and source are credited. 


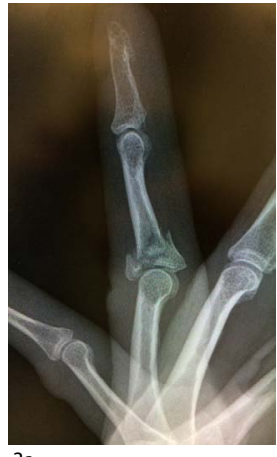

$3 a$
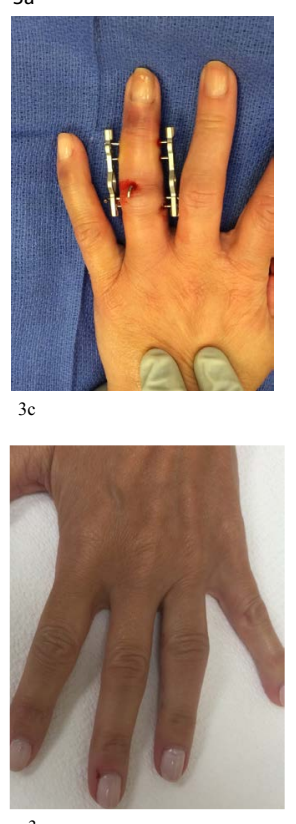

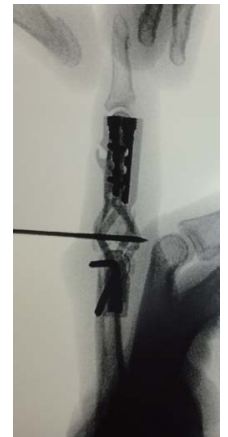

$3 b$
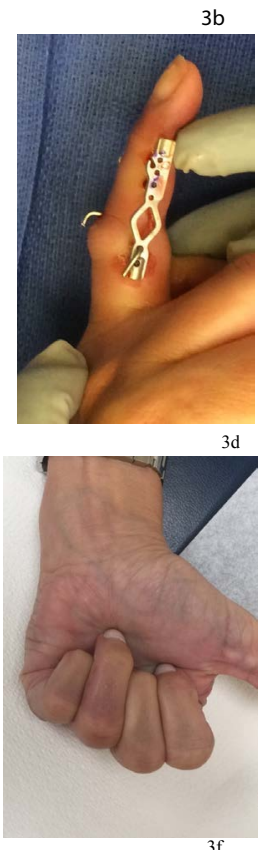

Figure 3: a) Lateral radiograph of a PIP pilon fracture. b-d) Intra-operative fluoroscopy and clinical photographs showing the DigiFix ${ }^{\circledR}$ external fixator in place. e \& f) Clinical photographs at 4 months post-op.
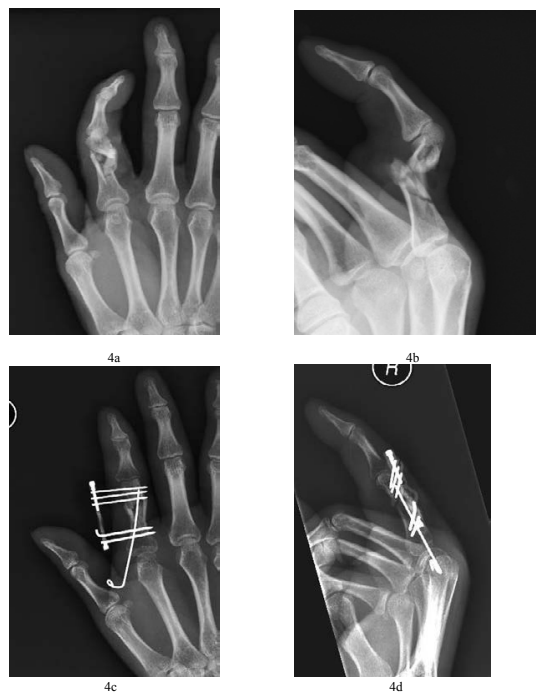

Figure 4: a) and b) Injury radiographs of a comminuted, open P1 fracture of the index finger. There was gross instability through the fracture. c \& d) DigiFix® application to permit PIP motion.
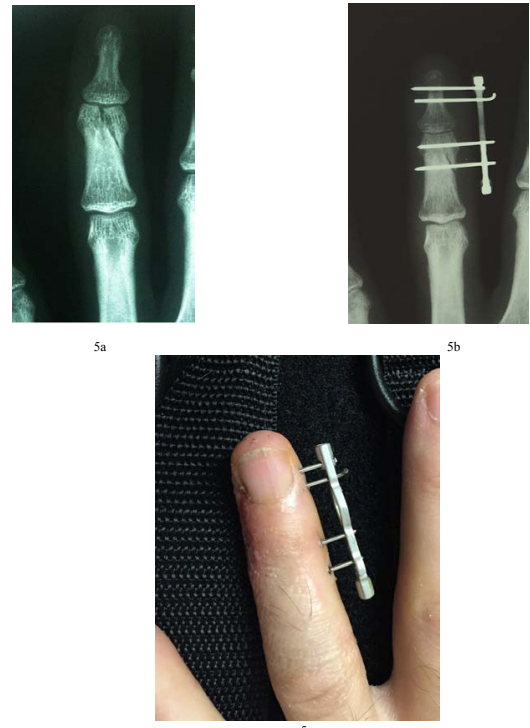

Figure 5: a) PA radiograph of a P2 condyle fracture with articular step-off and gapping. b) PA radiograph after application of the DigiFix ${ }^{\circledR}$ on the radial side of the finger. c) Corresponding clinical photograph with the DigiFix $\AA^{\circledR}$ in place.

she had painless active motion from $0^{\circ}-70^{\circ}$ at the PIP joint. At 4 months, the PIP motion improved to $0^{\circ}-90^{\circ}$ (Figure $3 \mathrm{e}$ and $3 \mathrm{f}$ ). Radiographs showed concentric reduction with preservation of the joint space.

\section{Case 3: Comminuted Phalangeal Shaft Fracture}

LF is a 52 year old right hand dominant male who injured his dominant index finger in a meat grinder. He sustained an open fracture of P1 (Figure $4 \mathrm{a}$ and $4 \mathrm{~b}$ ) and was taken to the operating room for irrigation and debridement and provisional fracture stabilization with $2 \mathrm{~K}$-wires. One of the K-wires was placed across the PIP to achieve distal purchase. Three weeks after the index procedure, the patient was taken back to the OR for fracture augmentation with the DigiFix ${ }^{\oplus}$, and the K-wire was backed out of the PIP to allow motion (Figure $4 \mathrm{c}$ and $4 \mathrm{~d}$ ). The external fixator was kept on for 6 weeks. At 2 months after the external fixator removal, the index finger PIP joint had active motion from $5^{\circ}$ $60^{\circ}$. Radiographs showed a healed fracture. The patient was continuing therapy at the time of this writing.

\section{Case 4: Intra-articular Condyle Fracture}

The patient is a 36-year-old police officer was injured in a carjacking incident. He sustained a lumbar burst and scapular body fracture, and a non-displaced ring finger unicondylar intra-articular DIP fracture. The ring finger fracture was initially treated with an alumaform splint. In follow-up, it was noted that the DIP was more radially deviated, and radiographs confirmed displacement of the fracture with gapping at the articular surface (Figure 5a). He was taken to the operative room at about 2 weeks post-injury for a percutaneous external fixation across the DIP joint with the DigiFix ${ }^{\oplus}$. A unilateral configuration was used and the fixator frame was applied in the static mode with distraction to reduce the articular fragment and restore neutral alignment in the coronal plane (Figure $5 \mathrm{~b}$ and $5 \mathrm{c}$ ).

At 3.5 weeks, the external fixator frame was removed but the two $\mathrm{K}$-wires in $\mathrm{P} 2$ were kept in place for another week. At 8 weeks post-op, the patient had $0-30^{\circ}$ active motion through the DIP without pain and had full flexion into the palm. 


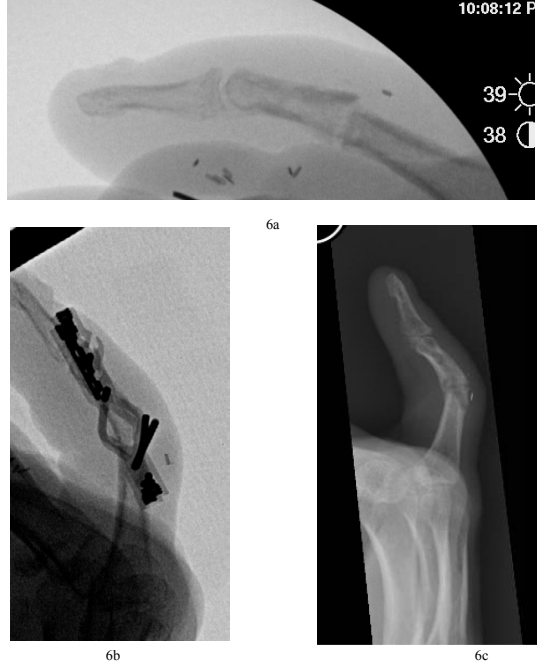

Figure 6: a) Intra-operative lateral fluoroscopic view of the small finger PIP fusion site. The PIP was in near full extension. b) After revision surgery with percutaneous application of the DigiFix ${ }^{\circledR}$ in compression mode. c) Lateral radiograph at 7 months after the revision surgery.

\section{Case 5: Interphalangeal Joint Arthrodesis}

The patient is a 44 year old right hand dominant laborer who had replantation of the amputated middle, ring and small fingers. Initial bony fixation was achieved with crossing K-wires. Attempted arthrodesis of the small finger PIP joint was done at the time of the initial surgery; however, there was a delayed union and loss of normal cascade due to the position of fusion (Figure 6a). Three months after the replantation, the patient was taken back to the operating room for revision of the small finger PIP arthrodesis. Because of concerns for vascular compromise, further soft tissue dissection and stripping were avoid by placing the DigiFix ${ }^{\oplus}$ external fixator percutaneously, without opening the joint. The position of fusion was corrected to $30^{\circ}$ of flexion in the sagittal plane (Figure $6 \mathrm{~b}$ ) and neutral in the coronal plane. The fixator was placed in the static mode and compression of the fusion site was performed through the Diamond portion of DigiFix ${ }^{\circ}$.

At 6 weeks, the fusion was clinically stable, and the fixator was removed in the office. At 7 months, the patient underwent staged tendon reconstruction of the ring and middle fingers. Radiographs showed bone bridging at the fusion site (Figure 6c). No further procedure was done for the small finger.

\section{Discussion}

An external fixator in its most basic form will stabilize a fracture and promote healing. The adjustability of a fixator, either to compress or distract, is advantageous to accommodate various injury patterns. External fixation of finger injuries has been previously described [1$3]$, however, most commercially available devices are not specifically designed for the hand, but are "miniaturized" from their larger counterparts to accommodate the small hand bones $[4,5]$. Particular to the hand, early concentric joint motion is crucial which most devices do not permit. As such, many surgeons improvise intra-operatively by bending $\mathrm{K}$-wires to fashion the desired effect of an external fixator [6,7].

Case 1 demonstrates the management of the PIP fracture-dislocation which remains difficult in most circumstances. The principles of treatment involve restoration of articular congruity and early mobilization of the joint to improve functional outcome [8]. In the author's opinion, these two principles are best achieved by a dynamic external fixator. The advantages of the DigiFix ${ }^{\star}$ over an improvised device are its ease of use, better tolerability by the patient, and the ability to adjust the fixator in the post-operative period.

Case 2 is similar to Case 1 in that the same treatment principles were employed. However in this case, the fixator was applied in the static mode for the first several weeks, followed by dynamization, a feature that is not readily available in the other commercial or improvised fixators. This feature allows the surgeon the flexibility to start motion when it is clinically appropriate without the need for post-operative splinting.

Cases 3 and 4 highlight the use of the DigiFix ${ }^{ø}$ on just one side of the finger (unilateral configuration) and in static mode for the duration of treatment. Fracture patterns will dictate the configuration of fixation and at times the fixator frame is only required unilaterally. This versatility allows patients to better tolerate the fixator.

Revision arthrodesis in the finger can be technically challenging due to scarred soft tissue envelope, compromised bone quality, and potential hardware issues. After replantation, the challenge is increased many folds. The ability to perform percutaneous fixation and provide compression across the fusion site, as shown in Case 5, makes a difficult case manageable. Furthermore, arthrodesis with an external fixator avoids hardware prominence or irritation problems.

\section{Conclusion}

External fixation of fingers, either dynamic or static, as described in this article is a useful technique to treat otherwise difficult problems. The cases presented demonstrate the versatility and effectiveness of the DigiFix ${ }^{\oplus}$ external fixator.

\section{References}

1. Kaleli T, Ozturk C, Ersozlu S (2003) External fixation for surgical treatment of a mallet finger. J Hand Surg Br 28: 228-230.

2. Dailiana Z, Agorastakis D, Varitimidis S, Bargiotas K, Roidis N, et al. (2009) Use of a mini-external fixator for the treatment of hand fractures. J Hand Surg Am 34: 630-636.

3. Hotchkiss R (1995) Treatment of complex fracture dislocation of the PIP joint with dynamic hinged external fixation. Procs ASSH 50th Annual Meeting, San Francisco, USA.

4. Technique Guide: Mini External Fixator. Stabilize the phalanges and metacarpals (1996) In: Catalog information. Synthes, USA

5. Hoffmann II Micro External Fixation System (2007) In: Catalog information. Stryker, USA.

6. McCulley SJ, Hasting C (1999) External fixator for the hand: a quick, cheap and effective method. J R Coll Surg Edinb 44: 99-102.

7. Walter FL, Papandrea RF (2011) A mini external fixator for hand and finger fractures constructed from readily available materials. Tech Hand Up Extrem Surg 15: 215-218.

8. Suzuki Y, Matsunaga T, Sato S, Yokoi T (1994) The pins and rubber traction system for treatment of comminuted intra-articular fractures and fracture dislocations in the hand. J Hand Surg 199: 98-107. 